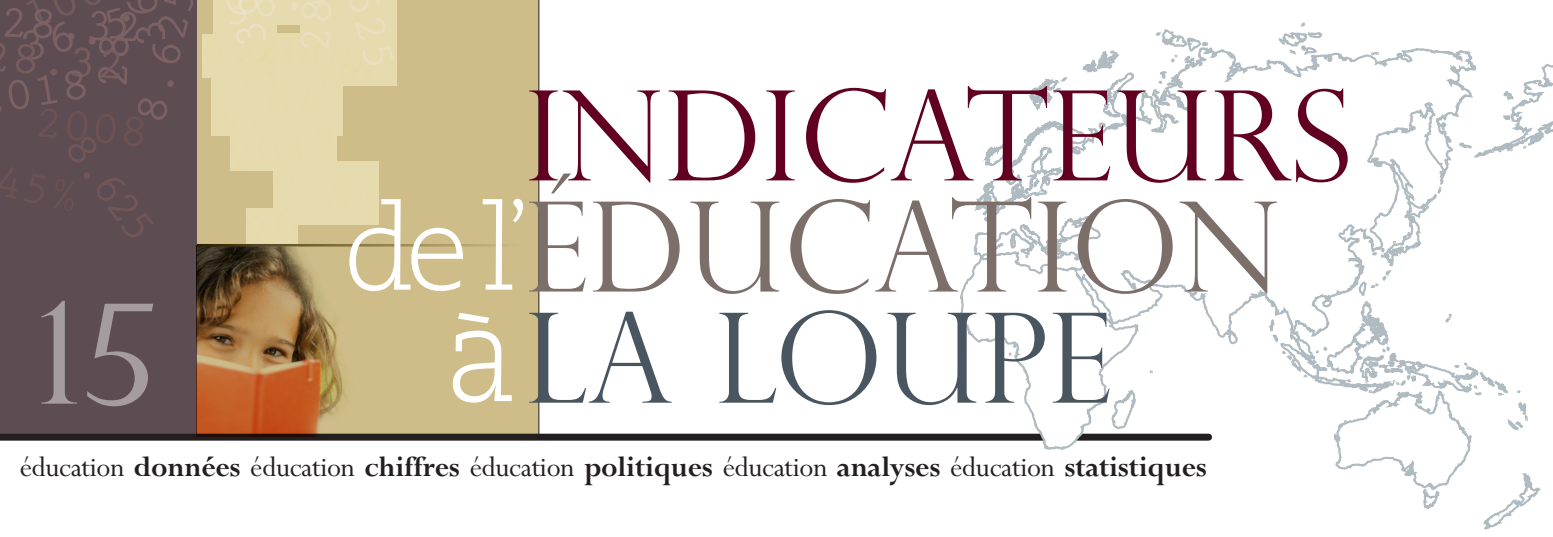

\title{
Comment le profil des étudiants de l'enseignement supérieur évolue-t-il ?
}

- En 2013, plus de 23 millions d'élèves des pays de l'OCDE et des pays du G20 se retrouveront pour la première fois sur les bancs de l'université.

- La nouvelle génération d'étudiants sera particulièrement variée, comprenant plus d'adultes et d'étudiants internationaux que jamais.

- Au cours des dernières décennies, les taux d'accès à l'université ont augmenté mais les inégalités persistent car ces chiffres sont le reflet du milieu socio-économique des élèves.

L'accès aux études supérieures est de plus en plus répandu...

Cette année, plus de 23 millions d'individus dans les pays de l'OCDE et les pays du G20 entameront un programme de niveau supérieur. Ils sont sur le point de s'engager dans de longues années d'études, espérant décrocher un diplôme mais aussi acquérir les connaissances et compétences nécessaires pour répondre aux besoins spécifiques de leur futur métier et de leur vie quotidienne. Leur performance au cours de ces études supérieures exercera un impact majeur non seulement sur leur propre avenir, mais aussi sur l'avenir de la société dans son ensemble.

L'accès aux études supérieures s'est fortement développé au cours des dernières décennies. Entre 1995 et 2011, les taux d'entrée à l'université ont augmenté de plus de 20 points de pourcentage en moyenne dans les pays de l'OCDE, passant de $39 \%$ à $60 \%$. Dans de nombreux pays, cette augmentation est due à la croissance de la demande, tandis que dans d'autres pays, elle est due à des changements structurels du système d'éducation (comme la mise en œuvre du Processus de Bologne). Dans le même temps, de nouveaux programmes ont été créés pour répondre aux évolutions des différents métiers auxquels préparent les études. Le vivier de candidats aux études supérieures s'est également étoffé et inclut désormais un bien plus grand nombre étudiants internationaux et d'étudiants plus âgés, ce qui a contribué à accroître les taux d'entrée.

Taux d'accès à l'enseignement supérieur (1995 - 2011)

On évalue à $60 \%$ la proportion de jeunes qui vont entamer un programme de niveau universitaire au cours de leur vie.

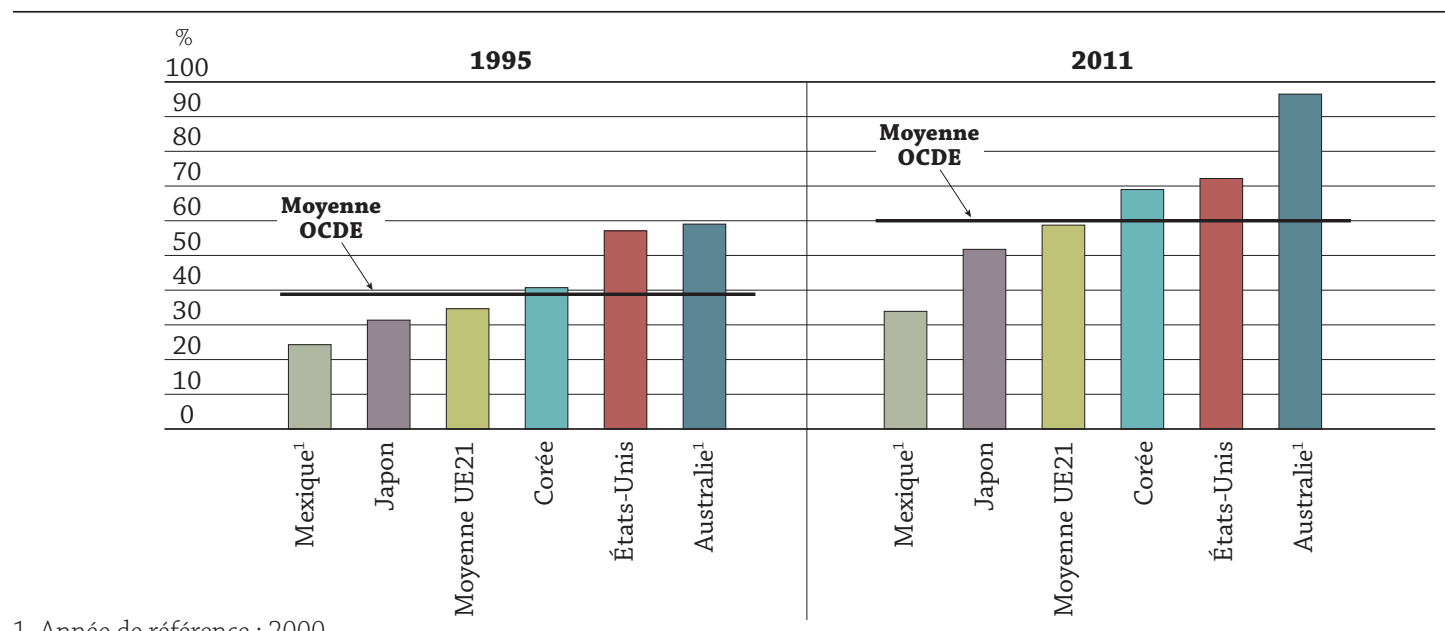

1. Année de référence : 2000.

Source : OCDE, Regards sur l'éducation 2013 : Les indicateurs de l'OCDE, indicateur C3 (www.oecd.org/edu/rse.htm). 


\section{... et le profil de l'étudiant type a évolué.}

Traditionnellement, les étudiants entraient à l'université juste après avoir terminé leur enseignement post-secondaire, et c'est toujours le cas dans de nombreux pays. Par exemple, en Belgique, au Japon et en Indonésie, l'âge moyen des nouveaux inscrits à l'université est 19 ans, voire moins. Cependant, l'âge moyen des nouveaux inscrits varie d'un pays à l'autre en raison de l'âge typique auquel les élèves terminent leurs études post-secondaires, de la capacité d'accueil des établissements et du coût d'opportunité d'une entrée sur le marché du travail avant d'avoir entamé des études supérieures. Par exemple, dans des pays tels que I'Islande, la Nouvelle-Zélande et la Suède, l'âge moyen des nouveaux inscrits est 25 ans ou plus. En moyenne, les étudiants des pays de l'OCDE et des pays du G20 auront 22 ans lorsqu'ils suivront leur premier cours de niveau supérieur et passeront 4.4 ans à étudier à temps plein.

Du point de vue de la différence entre les sexes, l'écart traditionnel s'est inversé, favorisant désormais les femmes. Ce n'est qu'en Arabie saoudite, en Indonésie, au Japon et au Mexique que les femmes sont moins nombreuses que les hommes. Cependant, plus le niveau d'enseignement augmente, plus l'écart se resserre : dans les programmes de recherche avancée, cet écart disparaît presque complètement.

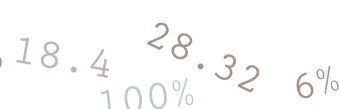

Par ailleurs, I'expansion rapide de l'enseignement supérieur dans les économies du G20 en pleine croissance a fortement modifié la répartition du vivier mondial de talents entre les pays. À I'heure actuelle, la Chine fait état de la proportion de nouveaux inscrits la plus importante, suivie des États-Unis et de I'Inde.

La nouvelle génération d'étudiants est également mobile du point de vue international. Le nombre d'étudiants internationaux a plus que doublé au cours de la dernière décennie. Aujourd'hui, parmi les nouveaux étudiants, quelque $4 \%$ quitteront le pays dont ils sont ressortissants pour aller étudier à l'étranger. En 2011, les proportions les plus importantes d'étudiants étrangers provenaient de la Chine, de la Corée et de l'Inde.

\section{Répartition dans le monde des étudiants étrangers de l'enseignement supérieur, par région d'origine (2011)}

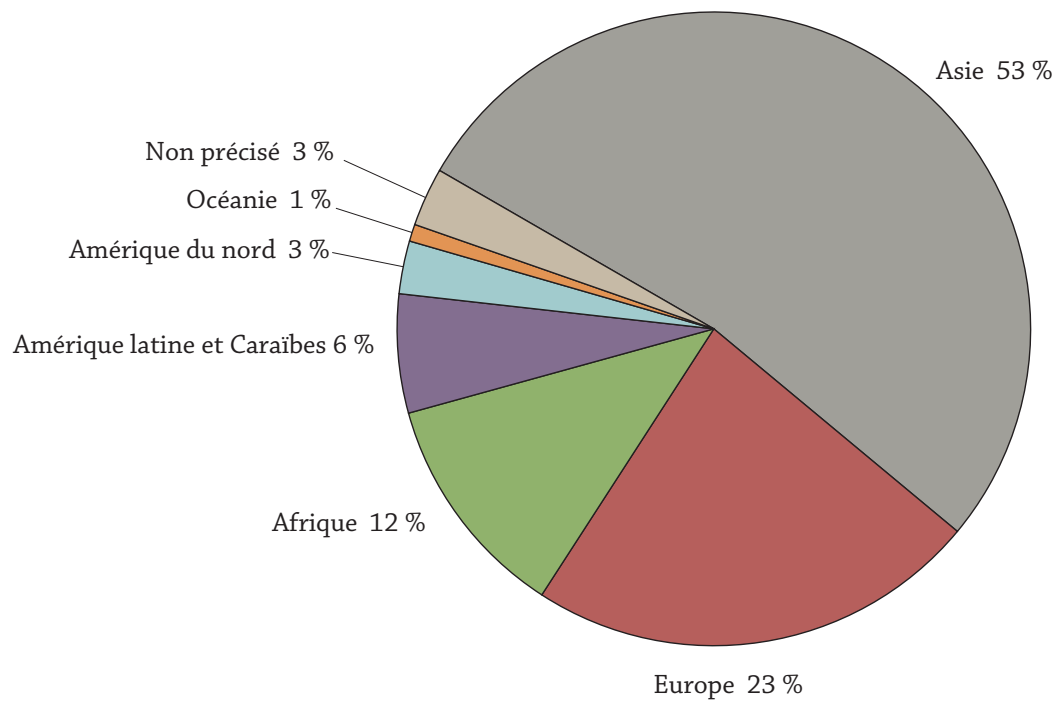

Source : OCDE, Regards sur l'éducation 2013 : Les indicateurs de l'OCDE, indicateur C4 (www.oecd.org/edu/rse.htm). 
Les étudiants asiatiques représentaient $53 \%$ des étudiants étrangers de l'enseignement supérieur dans des pays de I'OCDE et les pays du G20, et trois sur quatre d'entre eux étaient inscrits dans un pays de l'OCDE. La langue (qui peut constituer un obstacle ou au contraire une passerelle) est l'un des tout premiers critères dans le choix du pays d'études. Les pays anglophones sont les destinations les plus attractives mais de plus en plus de pays non anglophones commencent à proposer des cours en anglais afin de surmonter leur désavantage linguistique (voir OCDE, 2013).

\section{Égalité des chances?}

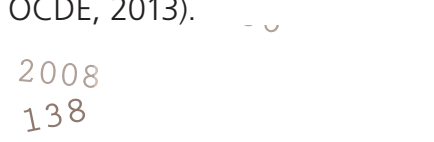

En général, plus leurs parents sont éduqués, plus les jeunes sont susceptibles d'entamer des études de niveau supérieur. En moyenne, les 20-34 ans provenant de familles très éduquées ont presque deux fois (1.9 fois) plus de chances de commencer des études supérieures que leurs pairs dont les parents sont moins éduqués. Cette tendance est particulièrement marquée au Portugal et en Turquie, où les jeunes de familles très éduquées sont plus de trois fois plus susceptibles de commencer des études universitaires (OCDE, 2012).

\section{Les matières scientifiques n'ont plus autant la cote qu'autrefois.}

Dans presque tous les pays, les domaines d'études les plus prisés sont les sciences, le commerce et le droit. En 2011, ces filières recevaient les proportions les plus importantes de nouveaux inscrits dans tous les pays sauf la Finlande, où l'ingénierie, la production et la construction occupaient la première place, et l'Arabie saoudite et la Corée, où les lettres, les sciences humaines, l'art et l'éducation l'emportaient.

\section{Répartition des nouveaux inscrits dans l'enseignement supérieur, par domaine d'études (2011)}

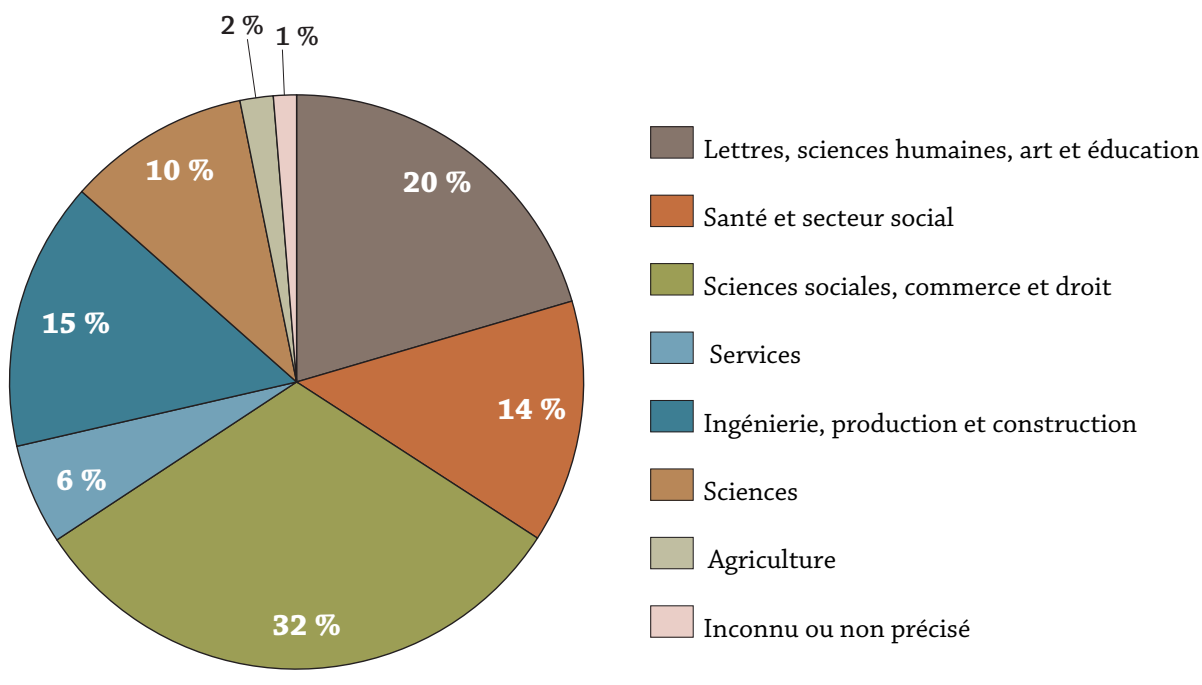

Source : OCDE, Regards sur l'éducation 2013 : Les indicateurs de l'OCDE, indicateur C3 (www.oecd.org/edu/rse.htm).

Les matières liées aux sciences - qui comprennent l'ingénierie, la production et la construction - sont moins populaires. En moyenne, seulement $25 \%$ des élèves choisissent cette filière, et les femmes y sont particulièrement peu représentées : en 2011, seulement $14 \%$ de femmes commençaient un programme de niveau supérieur dont le sujet était lié aux sciences, contre $39 \%$ d'hommes. Parmi les nouveaux inscrits, la proportion de femmes qui choisissent des matières liées aux sciences variait de $5 \%$ en Belgique et au Japon jusqu'à $19 \%$ en Grèce, en Indonésie, en Italie et au Mexique. Chez les hommes, les effectifs dans ces filières s'étalaient entre $18 \%$ en Argentine à $58 \%$ en Finlande. 


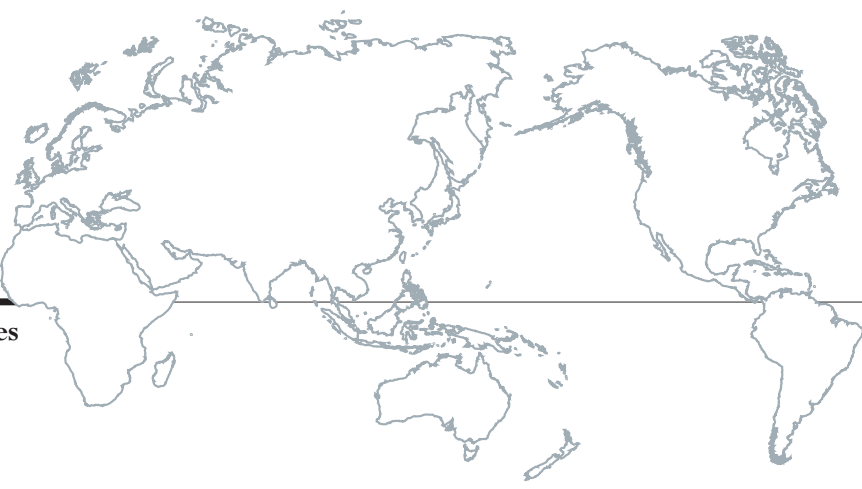

La plupart des étudiants de l'enseignement supérieur achèveront leurs études...

En moyenne, dans les pays de l'OCDE, environ $70 \%$ des étudiants qui commencent un premier programme de niveau supérieur cette année obtiendront un diplôme à ce niveau. Cependant, les taux d'obtention d'un diplôme varient fortement d'un pays de I'OCDE à l'autre. En Hongrie, en Norvège et en Suède, moins de $60 \%$ des étudiants qui commencent des études universitaires obtiendront leur diplôme, alors qu'en Australie, au Danemark, au Japon et en Turquie, ces taux d'obtention atteignent ou dépassent $80 \%$.

Les femmes sont davantage susceptibles que les hommes de décrocher un diplôme à la fin de leur cursus. Les taux d'obtention d'un diplôme s'établissent en moyenne à $74 \%$ chez les femmes et $65 \%$ chez les hommes. Ce n'est qu'en Allemagne, en Autriche, aux États-Unis et en Suède que l'écart entre les taux d'obtention d'un diplôme des hommes et des femmes reste au-dessous de cinq points de pourcentage.

\section{... mais pas tous.}

Lorsque l'on ne décroche pas son diplôme, cela ne signifie pas pour autant que les connaissances et compétences acquises au cours du cursus sont perdues ou non valorisables sur le marché de l'emploi. Obtenir une première expérience professionnelle peut aussi être utile aux individus plus tard dans leurs études. Par exemple, aux États-Unis et en Suède, il est plus courant que dans d'autres pays d'interrompre ses études supérieures avant de les avoir achevées, de travailler pendant quelque temps puis de reprendre ses études plus tard. Dans les pays dont le système est modulaire, comme la Suède, les étudiants reçoivent des crédits pour chaque cours qu'ils achèvent et ne perdent pas le bénéfice des modules validés avant d'avoir commencé à travailler.

Les étudiants peuvent aussi choisir de quitter les études avant d'obtenir leur diplôme car on leur propose des opportunités professionnelles intéressantes après seulement une année d'études. De même, certains étudiants plus âgés qui commencent un programme de niveau supérieur n'ont pas forcément l'ambition d'obtenir un diplôme. II s'agit plutôt pour eux de suivre quelques cours dans le cadre de l'apprentissage tout au long de la vie, ou bien d'étoffer leurs compétences.

\section{Références}

OCDE (2013), «Quels facteurs influencent la mobilité internationale des étudiants ? », Indicateurs de l'éducation à la loupe, $n^{\circ} 14$, Éditions OCDE.

OCDE (2012), Regards sur l'éducation 2012 : Les indicateurs de l'OCDE, Éditions OCDE.

Pour conclure Les établissements d'enseignement supérieur doivent non seulement répondre à la demande croissante de formation universitaire en augmentant le nombre de places, mais ils doivent aussi adapter les programmes et les méthodes d'enseignement pour satisfaire les besoins d'une génération d'étudiants de plus en plus diverse.

\begin{tabular}{l|l} 
Voir : & Consulter : \\
$\begin{array}{l}\text { www.oecd.org/ } \\
\text { edu/rse.htm }\end{array}$ & OCDE (2013), Regards sur l'éducation 2013: \\
& Les indicateurs de l'OCDE, Éditions OCDE. \\
&
\end{tabular}

Pour plus d'informations,
contacter :
Ignacio Marin
(Ignacio.Marin@oecd.org)
Corinne Heckmann
(Corinne.Heckmann@oecd.org)

